\title{
Consumo de alcohol en mujeres embarazadas atendidas en el Hospital Civil de Guadalajara Dr. Juan I. Menchaca, entre 1991 y 1998
}

\section{Introducción}

El consumo excesivo de alcohol por mujeres reviste un doble problema. Por una parte, es bien conocido que el alcoholismo, en hombres y mujeres, además de mermar la salud, afecta la

Si bien los efectos teratogénicos del alcohol fueron descritos desde hace más de 40 años, muchas mujeres en el mundo continúan consumiendo alcohol durante

el embarazo. Se realizó un análisis de archivo en el Hospital Civil de Guadalajara

Dr. Juan I. Menchaca a fin de conocer el porcentaje de mujeres que reportaron consumir alcohol durante el embarazo entre 1991 y 1998, su asociación con el consumo de tabaco y drogas ilícitas, así como variaciones de acuerdo a la edad.

Se encontró una prevalencia de $2.42 \%$ que se mantiene en los años analizados, el consumo más frecuente es el de alcohol asociado a tabaco y un efecto de la edad en los patrones de consumo donde la asociación alcohol- droga ilícita es más frecuente entre las mujeres más jóvenes.

Palabras clave: alcohol, embarazo, exposición alcohólica prenatal, adicciones, síndrome alcohólico fetal.

- Maestra en Ciencia del Comportamiento. Instituto de Neurociencias. CUCBA. Universidad de Guadalajara.

$\leftrightarrow$ Profesora-investigadora del Instituto de Neurociencias, CUCBA, y del Departamento de Estudios en Educación, CUCSH. Universidad de Guadalajara.

ematute@cencar.udg.mx vida social, que incluye aspectos familiares, laborales y educativos de quien lo padece y por la otra, las mujeres en edad fértil que consumen alcohol corren el riesgo de tener hijos con anomalías físicas e intelectuales. No obstante lo anterior, muchas mujeres consumen alcohol durante el embarazo. El objetivo de este trabajo es dar cuenta de los patrones de consumo de alcohol y su frecuencia en mujeres embarazadas que acuden al servicio de ginecología del Hospital Civil Juan I. Menchaca, uno de los hospitales de Guadalajara con mayor afluencia de pacientes. Para enmarcar este fenómeno, abordamos dos aspectos como antecedentes buscando la comprensión de este comportamiento. El primero de ellos, es sobre la prevalencia y morbilidad del consumo de alcohol 
en mujeres mexicanas y el segundo, sobre el efecto teratogénico del alcohol. Este trabajo pretende ser exploratorio, ya que son escasos los trabajos que se han realizado sobre este tema en población tapatía, y busca aportar las bases necesarias para indagar los motivos, situaciones de las mujeres para consumir alcohol durante el embarazo, así como la magnitud y frecuencia de los efectos de este consumo en los niños que nacen de ellas.

\section{Morbilidad del consumo de alcohol}

El abuso del alcohol es causa de un número importante de padecimientos físicos y de accidentes de todo tipo. Según el Manual Diagnóstico y Estadístico de los trastornos mentales, en su cuarta edición (DSM-IV), cerca de 90\% de los adultos en Estados Unidos ha probado el alcohol y aproximadamente $60 \%$ de hombres y $30 \%$ de mujeres se ha visto involucrado en alguna situación adversa relacionada con el abuso de éste, como serían accidentes de tráfico o bajas laborales (American Psychiatric Association, 2002). Para México, una proporción importante de la población de 12 a 65 años reporta haber presentado problemas asociados con su forma de beber; entre éstos, $3.4 \%$ de la población reportó haber tenido discusiones o peleas familiares, $2.2 \%$ problemas con las autoridades de tránsito y $0.8 \%$ reporta haber sido arrestado por haber conducido después de tomarse algunas copas (Consejo Nacional de Adicciones, 2002).

Con relación a la prevalencia de consumidores de alcohol en México, la Encuesta Nacional de Adicciones del 2002, realizada por el Consejo Nacional de Adicciones, reporta que poco más de 32.3 millones de personas entre 12 y 65 años de edad (46.3\%) han consumido bebidas con alcohol en el país, más los hombres (78.3\%) que las mujeres (46.4\%). De ellos, la mayoría eran bebedores en el momento de contestar la encuesta (61.1\% de los hombres y $34.2 \%$ de las mujeres). Sin

\section{2}


embargo, el consumo es poco frecuente, ya que sólo 7.04\% de la población reportó consumirlo al menos una vez por semana, más los hombres (13.2\%) que las mujeres (2.02\%). Particularmente en los hombres las ocasiones de consumo se asocian con grandes cantidades. Tanto los hombres como las mujeres tienden a consumirlo en su propia casa o en casa de otras personas.

No todas las personas que consumen alcohol sufren de trastornos relacionados con su consumo. En general, el consumo de alcohol de manera operacional se define como leve, moderado o alto (Abel, Krugel y Friedl, 1998). Los trastornos que pueden producirse por el uso de alcohol se relacionan con el consumo alto y son de dos tipos: de abuso y de dependencia (American Psychiatric Association, 2002). Estos trastornos se presentan principalmente en hombres. Con relación a la población femenina, los índices de abuso/ dependencia muestran pocas diferencias al comparar las encuestas de 1998 y 2002. En 1998, 4\% de las mujeres reportaron beber cinco copas o más por ocasión de consumo una vez al mes o más frecuentemente, en tanto que para 2002, la proporción que reportó este patrón de consumo fue de 5.3\%. Sin embargo, los índices de crecimiento en el abuso/dependencia entre las adolescentes menores de edad se incrementó significativamente de $0.2 \%$ a $0.8 \%$ en este periodo. Con relación al índice de dependencia, 90\% de las mujeres beben con niveles de bajo riesgo o son abstemias, en tanto que 14,703 mujeres tienen dependencia severa y requieren de tratamiento especializado; 231,300 mujeres tienen dependencia moderada o leve o problemas con el manejo del alcohol y pueden beneficiarse con programas de intervención breve. Más aún, el porcentaje de mujeres que buscan ayuda por problemas en el uso del alcohol aumentó en $2 \%$ de 2001 a 2002 (Secretaría de Salud Jalisco: Sistema de Vigilancia Epidemiológica de las Adicciones, 2002). 
Con respecto a la edad de las mujeres que consumen alcohol, de acuerdo a esta misma encuesta, el mayor porcentaje de bebedoras (40.35\%) se ubica en el rango de 18 a 29 años de edad, lo cual coincide con la edad para tener hijos. Más aún, el mayor porcentaje de mujeres (3\%) que reportan consumo de alcohol diario, casi diario o de una a cuatro veces por semana corresponde a este mismo rango de edad.

Así, en México, no sólo se observa un aumento en el consumo de alcohol por las mujeres, sino que la frecuencia y cantidad de ingesta de alcohol es mayor en las mujeres de 18 a 29 años de edad, que son aquéllas que están en edad de procreación. El hecho de que las mujeres estén presentando mayores problemas con el abuso del alcohol, además de las consecuencias a nivel emocional, social y familiar que esto provoca - pues se sabe que un miembro alcohólico en la familia ocasiona una disfuncionalidad importante dentro de ésta - incrementa el riesgo de que aquéllas en edad fértil, puedan exponer a sus hijos al alcohol antes de nacer con todas las consecuencias educativas y de salud que de esto se derivan.

\section{Efectos teratogénicos del alcohol}

Las mujeres embarazadas que consumen alcohol corren el riesgo de que éste genere un desarrollo anormal y malformaciones congénitas en el bebé que esperan. Estos efectos teratogénicos del alcohol fueron descritos por primera vez en Francia en 1967 por Lemoine y sus colaboradores al analizar hijos nacidos de madres alcohólicas crónicas (Picherot, 2008). Más tarde, en 1973, Jones y Smith reportaron características similares en niños expuestos al alcohol en su vida prenatal y acuñaron el término síndrome alcohólico fetal (SAF), el cual es reconocido actualmente como una entidad clínica (Streissguth, 2001). Estos investigadores hicieron énfasis en los problemas de crecimiento y en las anomalías 
faciales observadas en estos niños; al paso de los años se han agregado otras características para la definición de este síndrome. El Instituto de Medicina de la Academia Nacional de Ciencias de Estados Unidos establece como criterios para realizar el diagnóstico de este síndrome (Stratton, Howe, Battaglia, 1996) la presencia de historia de exposición alcohólica prenatal acompañada de evidencia de alteraciones faciales características, de retardo en el crecimiento y de alteraciones en el desarrollo del sistema nervioso central.

Existen además otros niños que si bien estuvieron expuestos al alcohol en vida intrauterina, sólo cumplen con algunas de las características del SAF (especialmente las faciales) y son diagnosticados como niños con efectos alcohólicos fetales. En ambas situaciones se pueden presentar trastornos cognitivos graves (Mattson et al., 1998). La incidencia del SAF y de casos considerados como efectos alcohólicos fetales, se ha estimado en 9.1/1000 nacimientos en Estados Unidos (Sampson et al., 1997).

Los resultados de los estudios sobre los efectos del alcohol en el desarrollo cognitivo de los niños que fueron expuestos a él en vida intrauterina han mostrado que los sujetos con SAF tienden a presentar con mayor frecuencia un cociente intelectual (CI) limítrofe, mientras que en los sujetos con efectos fetales del alcohol es común encontrar un CI normal (Connor et al., 2000; Carmichael Olson et al., 1998); aunque hay autores que no encuentran diferencias al respecto (Schonfeeld, Mattson y Lang, 2001; Mattson et al., 1998). En cuanto a habilidades específicas, se han reportado alteraciones en diferentes tipos de memoria (Carmichael Olson et al., 1998; Mattson y Riley, 1999; Connor et al., 2000; Willford et al., 2004), en las habilidades numéricas (Kopera-Frye, Dehaene y Streissguth, 1996) y visoespaciales (Carmichael Olson et al., 1998; Willford et al., 2004) así como en las funciones ejecutivas (Carmichael Olson et al., 1998; Connor et al., 2000; Schonfeld, Mattson y Lang, 2001). Los trastornos 
cognitivos ocasionados por la exposición alcohólica prenatal requieren muchas veces de una atención especializada para su rehabilitación y pueden tener impacto durante toda la vida del individuo (Streissguth y O'Malley, 2000).

Hasta ahora no se ha identificado un perfil cognitivo único y característico de los sujetos con exposición alcohólica prenatal. Lo anterior puede estar relacionado a que, por una parte, los efectos deletéreos del alcohol están influenciados por muchas otras variables y por otro, al uso de diferentes metodologías en el momento de la evaluación, lo que conlleva a resultados poco comparables.

Las anomalías faciales que caracterizan al SAF se pueden identificar claramente en edades tempranas (antes de los siete años) y parecen atenuarse a lo largo del desarrollo (Streissguth, Bookstein y Barr, 1996). En lo que se refiere al retardo en el crecimiento, se ha encontrado que los niños tienden a recuperar el peso normal, aunque pudieran presentar baja estatura (Habbick et al., 1998).

Una de las preguntas que sigue sin resolverse es si existe algún consumo de alcohol "seguro" durante el embarazo (Jacobson y Jacobson, 1994); hasta el momento la respuesta parece ser no. La dificultad para responder esta cuestión reside en que el daño que puede causar el alcohol al feto en desarrollo está influido por otras variables tales como: la edad de la madre, el número de gesta, el estado nutricional y el metabolismo de la madre, el periodo de gestación en el que se dio la exposición, la variación en la vulnerabilidad de las diferentes estructuras cerebrales, el patrón de consumo del alcohol, la ingesta de otras sustancias tóxicas, etcétera (Maier y West, 2001).

A este respecto existen autores que han fallado en encontrar alteraciones provocadas tras un bajo consumo de alcohol, mientras que hay otros que sí las reportan (Sood et al., 2001). En este punto se plantea otro problema importante: no existe un consenso acerca de las definiciones del consumo 
bajo, moderado o alto, aun cuando se ha demostrado que los médicos tienen un concepto similar acerca de la definición de los diferentes niveles de consumo (Abel, Kruger y Friedl, 1998). El hecho de que no exista una descripción formal sobre la cantidad de alcohol consumido por la madre para cada nivel de consumo resta claridad a los resultados cuando se indaga sobre el efecto de la exposición alcohólica prenatal sobre el niño.

En México, la Encuesta Nacional de Adicciones (ENA) (Secretaría de Salud et al., 1998), reportó que el porcentaje de mujeres consumidoras de alcohol en 1988 fue de 36.1, con un aumento considerable para 1998 cuando fue de $49.7 \%$. El incremento más importante se dio en el rango de edad de 50 a 65 años (de $24.4 \%$ a $44.6 \%$ ), seguido del de $40-49$ años (36.5\% a 46.6\%), en tercer lugar en el de 30 a 39 años (de $40.8 \%$ a $47.8 \%$ ), y finalmente, en el rango de 18 a 29 años (de $38.6 \%$ a $44.6 \%$ ). Los datos anteriores enfatizan la alta frecuencia de consumo de alcohol en mujeres en edad reproductiva y, derivado de ello, el riesgo en que se encuentran los niños de sufrir efectos adversos en su desarrollo ante dicho consumo.

El objetivo del presente trabajo es conocer la prevalencia de consumo de alcohol durante el embarazo en mujeres que dieron a luz en un periodo similar al reportado en el análisis de tendencias de la ENA y su variación con la edad. De la misma manera, quisimos conocer los patrones de consumo, es decir, la asociación del consumo de alcohol al del tabaco o drogas en los diferentes años analizados. Por último, nos interesó saber si la edad de la madre está relacionada con patrones de consumo distintos.

\section{Material y métodos}

Se trata de un estudio exploratorio y retrospectivo realizado a partir de la revisión de los libros de registro de 
nacimientos atendidos de 1991 a 1998 en el Hospital Civil de Guadalajara, Dr. Juan I. Menchaca. Éste es un hospitalescuela en el que reciben atención personas procedentes de todo el estado de Jalisco e incluso de estados vecinos, a un bajo costo. Es en este hospital donde se registra el mayor número de nacimientos de la ciudad. Se analizaron los registros de esos años ya que este estudio es la base de otro en el que se contempla analizar el efecto a largo plazo de la exposición prenatal al alcohol.

En el Departamento de Neonatología de este hospital se consigna la información de los recién nacidos y se vacía en un formato que es llenado a mano por el residente que atiende el parto, dicho formato contiene datos referentes a la madre y al recién nacido. Se revisaron un total de 78,871 registros. Para incluir un caso en la base, éste debería contar con los siguientes datos: edad de la madre, fecha de nacimiento del niño, presencia o ausencia de consumo de alcohol durante el embarazo, de tabaco y de otras drogas. Estos datos (a excepción de la fecha de nacimiento del niño) son reportados por la madre.

Para el análisis de los datos se utilizó el programa estadístico SPSS. Se analizaron frecuencias en números absolutos y porcentajes del consumo de alcohol y su asociación con tabaco o con drogas ilegales. Las comparaciones se realizaron por año, por tipo de consumo y por edad.

\section{Resultados}

I. Prevalencia del consumo de alcohol en mujeres embarazadas entre 1991 y 1998

Como puede observarse en la tabla 1 , del total de registros revisados $(78,871)$, encontramos una prevalencia de consumo de alcohol durante el embarazo de $2.42 \%(1,909$ casos). Se observa, además, que el número de registros de nacimiento se duplicó del año 1991 al de 1998; de igual

\section{8}


forma, el número de mujeres que confirmó haber consumido alcohol y su porcentaje también mostraron un incremento; sin embargo, éste no es estadísticamente significativo $(\mathrm{r}=$ $.429, \mathrm{p}<.29)$.

\section{Tabla 1}

Número de registros de nacimiento por año, número de madres que reporta consumo de alcohol durante el embarazo y porcentaje de éstas

\begin{tabular}{cccc} 
Año & $\begin{array}{c}\text { Número de } \\
\text { registros por año }\end{array}$ & $\begin{array}{c}\text { Número de registros con } \\
\text { reporte de consumo de alcohol }\end{array}$ & Porcentaje \\
1991 & 6702 & 132 & 1.97 \\
1992 & 6746 & 149 & 2.21 \\
1993 & 7490 & 167 & 2.23 \\
1994 & 9032 & 260 & 2.88 \\
1995 & 8663 & 193 & 2.23 \\
1996 & 12070 & 310 & 2.57 \\
1997 & 14395 & 313 & 2.17 \\
1998 & 13773 & 385 & 2.80 \\
Total & 78871 & 1909 & 2.42 \\
\hline
\end{tabular}

2. Asociación del consumo de alcohol con el consumo de tabaco y drogas ilícitas.

a) Análisis global

Además de calcular el porcentaje de consumo de alcohol reportado, se buscó la presencia de consumo de alcohol asociado con el uso de tabaco y/o de otras drogas en la población. Entre los 1,909 casos se detectaron cuatro patrones de consumo: sólo alcohol; alcohol y tabaco; alcohol y alguna droga ilícita (pero no tabaco) y alcohol, tabaco y droga.

Como puede observarse en la tabla 2, el consumo más frecuente es la asociación de alcohol y tabaco, seguido por sólo alcohol. Las asociaciones de alcohol, tabaco y droga y de alcohol y droga se presentan en un porcentaje inferior a 
los dos patrones de consumo anteriores. En 13.89\% de los casos, la información estaba incompleta por lo que no se pudo precisar el patrón de consumo en ellos.

\section{Tabla 2}

Número de casos de cada patrón de consumo

$y$ porcentaje que representa

\begin{tabular}{lrc}
\hline Patrón de consumo & $N$ & Porcentaje en que se presenta \\
\hline Alcohol y tabaco & 799 & 41.86 \\
Sólo alcohol & 703 & 36.84 \\
Alcohol, tabaco y droga & 121 & 6.35 \\
Alcohol y droga & 20 & 1.06 \\
Incompletos & 265 & 13.89 \\
Casos totales & 1909 & 100.00 \\
\hline
\end{tabular}

\section{b) Análisis por año}

En todos los años, el patrón menos frecuente es el de alcohol y droga ilícita, seguido del de alcohol, tabaco y droga ilícita; los otros dos patrones, sólo alcohol y alcohol asociado a tabaco, son los más frecuentes y se presentan en proporciones similares en los diferentes años analizados $\left(\mathrm{X}^{2}=16.918\right.$, $\mathrm{gl}=10, \mathrm{p}<0.76$ ) con una discreta tendencia al predominio de la asociación de alcohol y tabaco ante la disminución de sólo alcohol, en el patrón de consumo en los dos últimos años analizados, 1996 y 1998, así como un aumento en la asociación de alcohol, tabaco y droga ilícita (tabla 3).

\section{Variación de patrón de consumo de acuerdo a la edad}

La media de edad de las mujeres que reportaron haber consumido alcohol durante su embarazo es de 24.96 años. Como puede observarse en la figura 1, la media de edad de las mujeres que incluyeron drogas ilícitas en su patrón de consumo es significativamente menor a la de aquellas que no lo hicieron (21.47 vs 25.25 años) ( $p<.001$ ). 


\section{Tabla 3}

Porcentaje en el que se presenta cada patrón de consumo por año

\begin{tabular}{ccccc} 
Año & Sólo alcohol & $\begin{array}{c}\text { Alcoholy } \\
\text { tabaco }\end{array}$ & $\begin{array}{c}\text { Alcohol y droga } \\
\text { Alcohol, tabaco } \\
\text { y droga }\end{array}$ \\
1991 & 45.1 & 45.9 & 0.8 & 8.2 \\
1993 & 53.7 & 37.0 & 3.7 & 5.6 \\
1994 & 45.6 & 47.5 & 0.4 & 6.6 \\
1995 & 47.3 & 46.7 & 1.6 & 4.3 \\
1996 & 41.2 & 50.6 & 1.3 & 6.8 \\
1998 & 38.4 & 50.5 & 0.3 & 10.8 \\
\hline
\end{tabular}

Nota: no se tomaron en cuenta los años 1992 y 1997, ya que había un porcentaje significativo de casos incompletos: $37.6 \%$ y $41.9 \%$, respectivamente.

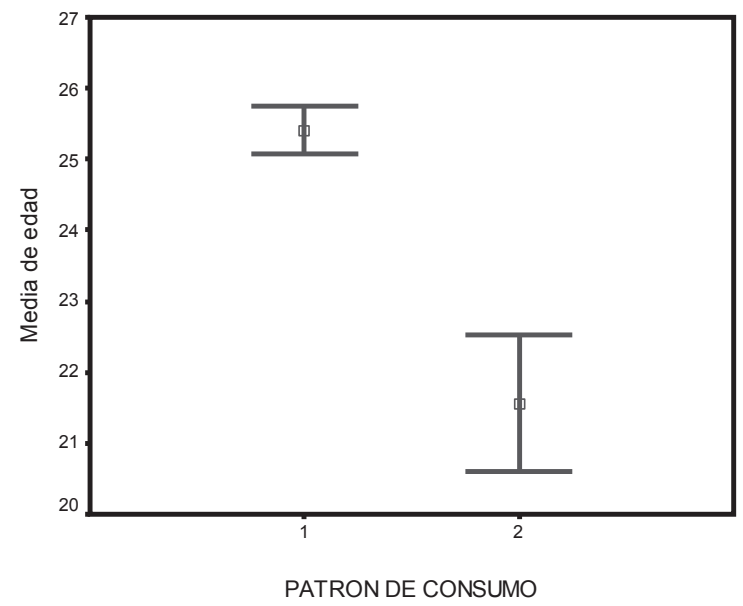

Figura I. Media de edad y dos errores estándar para todos los años analizados según patrón de consumo: (I) sin reporte de consumo de droga ilícita, (2) con reporte de consumo de droga ilícita.

En la figura 2 puede observarse que esta diferencia en el patrón de consumo con relación a las medias de edad es constante en todos los años, siendo significativa en 1993 $(p<.001), 1994(p<.005)$ y $1996(p<.001)$. 


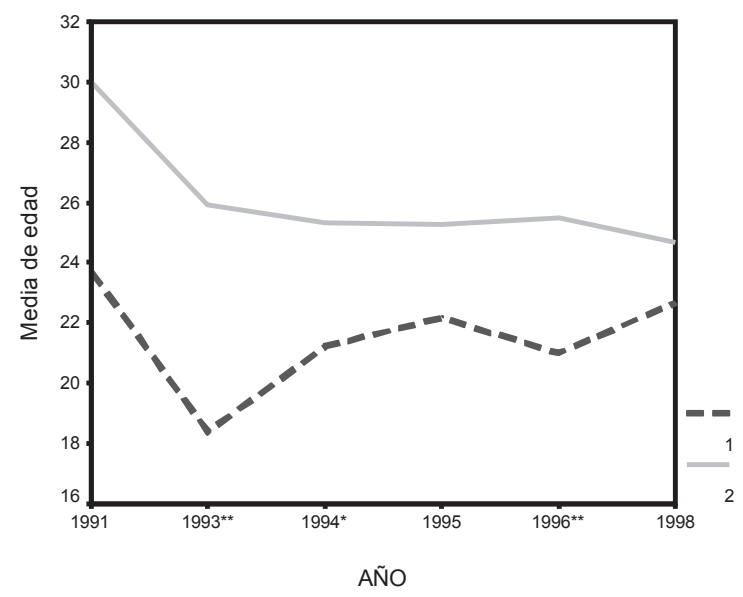

Figura 2. Media de edad por año, según patrón de consumo: I) con reporte de consumo de droga ilícita y 2 ) sin reporte de consumo de droga ilícita $(*=p<.005, * *=p<.001)$.

\section{Discusión}

El propósito de este trabajo fue conocer la prevalencia del consumo de alcohol durante el embarazo en mujeres que dieron a luz de 1991 a 1998 en un hospital público de Guadalajara, Jalisco, así como determinar las características del patrón de consumo y su relación con la edad.

En nuestro estudio, 2.41\% de mujeres reportó haber consumido alcohol durante el embarazo, porcentaje que es menor al promedio de los reportes de 1991 a 1998 hechos por el Centro de Control y Prevención de Enfermedades de Estados Unidos, que fue de 13.2\% (Centers for Disease Control and Prevention, 2002).

Si bien la Encuesta Nacional de Adicciones (1998) reporta un aumento significativo en el consumo de alcohol de las mujeres de 1988 a 1998, esta tendencia no resulta significativa en nuestra población de estudio. Esto podría explicarse por la existencia de una tendencia natural a 
dejar de consumir alcohol durante el embarazo reportada por Jacobson et al. (2002), que alcanza hasta 80\% según el estudio realizado en mujeres de bajos ingresos por Ockene et al. (2002), pero vuelve a su nivel habitual pre-embarazo después del parto (Jacobson et al., 2002). Si bien la validez de la información proporcionada por las mujeres cuando la encuesta es realizada en el momento del embarazo, ha sido cuestionada por algunos autores, quienes han señalado que los reportes hechos aproximadamente cinco años después del parto son más confiables, ya que en estos últimos se ha encontrado una mayor relación con los trastornos considerados marcadores del SAF que en aquellos realizados durante el embarazo (Ernhart et al., 1998). Sin embargo, Jacobson y col. (Jacobson et al., 2002) han encontrado que, aun cuando las mujeres reportan mayor consumo en la entrevista retrospectiva, el nivel de consumo informado por las mujeres de su estudio en la entrevista antes del nacimiento de sus hijos tuvo mayor correlación con los déficit neurocomportamentales relacionados al alcohol tales como: desempeño bajo en la prueba de Bayley, pobre nivel de juego, baja velocidad del procesamiento en la prueba Fagan II y elevado tiempo de reacción en el paradigma de expectación visual, y que incluso estos déficit no habrían sido detectados utilizando los datos de la entrevista restrospectiva.

En nuestro estudio, los patrones de consumo más frecuentes fueron "sólo alcohol" y "alcohol y tabaco", el reporte de consumo de droga fue el menos frecuente en todos los años. Otro dato relevante es que la media de edad de las mujeres que incluyeron droga en su patrón de consumo es significativamente menor a la de las mujeres que no reportaron consumo de drogas. Es probable que la mayor frecuencia de este patrón de consumo en las más jóvenes refleje el incremento en la proporción de usuarias de drogas, el cual se triplicó entre 1993 y 1998 especialmente en el 
grupo de 12 y 17 años de edad (Secretaría de Salud, 1998; Rosovsky, 1999).

Las encuestas nacionales de adicciones (ENA) se realizan cada cinco años y representan la fuente principal de información acerca de los problemas de consumo de sustancias en México. En la ENA de 1988, se incluyeron preguntas para las mujeres que habían estado embarazadas al menos una vez en su vida, con la finalidad de obtener información acerca del consumo de alcohol durante el embarazo y realizar estimaciones sobre éste y el riesgo de bajo peso y de nacimientos pre-término, entre otros (Borges et al., 1993). Si bien se obtuvieron datos interesantes, la encuesta no estaba diseñada especialmente para obtener información precisa sobre exposición alcohólica prenatal y no se dispone de una metodología formal para conocer los índices de consumo de alcohol durante el embarazo en las mujeres de México.

En la Encuesta Nacional de Adicciones realizada en 2002, se observa que $25 \%$ de la población urbana femenina de 12 a 17 años reportó haber consumido alcohol en los doce meses previos al estudio, mientras que así lo hizo $42.7 \%$ de las mujeres adultas, de 18 a 65 años. Los resultados de esta encuesta muestran una tendencia ascendente en el porcentaje de adolescentes consumidoras de alcohol, el cuál pasó de $18 \%$ en 1998 a $25 \%$ en 2002 , a la vez que el porcentaje de mujeres consumidoras adultas se mantuvo estable entre estos años (Secretaría de Salud, 2002).

Aunque, en comparación con otros países, el consumo de alcohol de las mujeres mexicanas, según las encuestas, no es muy alto y el índice alcoholismo es aún menor (Secretaría de Salud, 2002), hay varios datos que justifican la necesidad de difundir más información sobre los riesgos del consumo de alcohol durante el embarazo y de promover la abstinencia de su consumo durante este periodo. Entre éstos, la tendencia ascendente observada en este trabajo del porcentaje de mujeres que reportan consumo de alco- 
hol durante el embarazo y el aumento en el porcentaje de mujeres adolescentes consumidoras de alcohol reportado en la ENA, la cual es una población con alta tasa de fertilidad. Además, se debe considerar el hecho de que no se conoce un umbral de consumo seguro para la mujer embarazada y su hijo.

\section{Reflexiones finales}

Los resultados obtenidos en nuestra investigación abren un espacio para conocer en México la situación actual de consumo de alcohol y de otras substancias teratogénicas, lícitas o ilícitas por las mujeres embarazadas. En efecto, el contrastar nuestros datos con los correspondientes a la década actual recogidos en el archivo del mismo hospital aquí analizado, facilitará el conocimiento de la condición actual de este problema y su evolución en estos últimos 20 años.

Consideramos que en nuestro país es necesario implementar metodologías específicas para detectar el consumo de alcohol en mujeres embarazadas, así como realizar más investigación sobre el tema, con el fin de facilitar el diseño de medidas preventivas y a fin de reducir la incidencia de nacimientos de niños que requieran de manera prolongada atención educativa, médica y psicológica especializada.

\section{Agradecimientos}

Agradecemos especialmente la ayuda brindada por el doctor Gustavo Orozco, jefe del Departamento de Neonatología del Nuevo Hospital Civil de Guadalajara, "Dr. Juan I. Menchaca", así como la colaboración de Sara Dolores Núñez, por las facilidades prestadas para la revisión de los registros.

Fecha de recepción: 19 de enero de 2009.

Fecha de aceptación: $1^{\circ}$ de junio de 2009. 
Bibliografía

Abel, E. L, Kruger, M. L y J. Friedl (1998), How do physicians define "light", "moderate” and "heavy drinking"?, Alcoholism Clinical and Experimental Research, 22(5): 979-984. American Psychiatric Association (2002), Manual diagnóstico y estadístico de los trastornos mentales. Texto revisado (DSM-IV-TR). Barcelona, España, Masson.

Borges, G., López-Cervantes, M., Medina-Mora, M., TapiaConyer, R. y F. Garrido, (1993), "Alcohol Consumption, Low Birth Weight, and Preterm Delivery in the National Addiction Survey (Mexico)", International Journal of Addiction, 28(4): 355-368.

Carmichael Olson, H., Feldman, J. J., Streissguth, A. P., Sampson, P. D., F. L. Bookstein (1998), "Neuropsychological Deficits in Adolescents with Fetal Alcohol Syndrome: Clinical Findings", Alcoholism Clinical and Experimental Research, 22(9): 1998-20I2.

Centers for Disease Control and Prevention (2002), "Alcohol use among women of childbearing age- United States, I99I-1999", MMWR, 5 I (I3): 273-276.

Connor, P. D., Sampson, P. D., Bookstein, F. L., Barr, H. M. y A. P. Streissguth (2000), "Direct and Indirect Effects of Prenatal Alcohol Damage on Executive Function", Developmental Neuropsycholy, 18(3): 33I-354.

Ernhart, C. B., Morrow-Tlucak, M., Sokol, R. J., S. Martier (1988). Underreporting of alcohol use in pregnancy. Alcoholism Clinical and Experimental Research, 12: 506$5 \mathrm{II}$.

Habbick, B. F., Blakley, C., Houston, S., Snyder, R. E., Senthilselvan, A., J. L. Nanson (1998), "Bone Age and Growth in Fetal Alcohol Syndrome", Alcoholism Clinical and Experimental Research, 22(6): |3 | 2-1316.

Jacobson, S. W., Chiodo, L. M., Sokol, R. J., J. L. Jacobson (2002), "Validity of maternal report or prenatal alcohol, cocaine, and smoking in relation to neurobehavioral outcome”, Pediatrics, 109(5): 815-825. 
Jacobson, J. L. y S. W. Jacobson (1994), "Prenatal alcohol Bibliografía exposure and neurobehavioral development, where is the threshold?", Alcohol Research \& Health, I8(I): 30.

Jones, K. L. y D. W. Smith (1973), "Recognition of the fetal alcohol syndrome on early infancy”, Lancet, 3; 2 (7836): 999-100I.

Kopera-Frye, K., Dehaene, S. y A. P. Streissguth (1996), "Impairments of number processing induced by prenatal alcohol exposure", Neuropsychologia, 4(I2): I I871196.

Maier, S. E. y J. R. West (200I), Drinking Patterns and Alcohol-Related Birth Defects. Alcohol Research \& Health, 25(3): I68-I74.

Mattson, S. N. y E. P. Riley (I999), "Implicit and explicit memory functioning in children with heavy prenatal alcohol exposure", Journal of Clinical and Experimental Neuropsychology, 5: 462-47I.

Mattson, N. L., Riley, E. P., Gramling, L., Delis, D. C. y K. L. Jones (1998), "Neuropsychological comparison of Alcohol-Exposed Children with or without physical features of Fetal Alcohol Syndrome”, Neuropsychology, I2(I): I46I53.

Ockene, J.K., Y. Ma, J. G. Zapka, L. A. Pbert, K. V. Goins y A. M. Stoddard (2002), "Spontaneous Cessation of Smoking and Alcohol Use Among Low-Income Pregnant Women", American Journal of Preventive Medicine, 23(3): I50-I55.

Picherot, G. (2008), "Poetopathie alcoolique: à propos de Paul Lemoine”, Archives de Pédiatrie, I5(5): 506.

Rosovsky, H. (1999), El consumo de drogas en México: diagnóstico, tendencias y acciones. México, DF, Secretaría de Salud-Consejo Nacional contra las Adicciones.

Sampson, P. D., A. P. Streissguth, F. L. Bookstein, R. E. Little, S. K. Clarren, P. Dehaene, J. Hanson y J. M. Graham (1997), "Incidence of Fetal Alcohol Syndrome and 
Bibliografía

Prevalence of Alcohol-Related Neurodevelopmental Disorder", Teratology, 56: 317-326.

Schonfeld, A. M., S. N. Mattson y A. R. Lang (200I), "Verbal and nonverbal fluency in children with heavy prenatal alcohol exposure", Journal of Studies on Alcohol, 62(2): 236-246.

Secretaría de Salud, Subsecretaría de Salud y Control de Enfermedades, Dirección General de Epidemiología, Instituto Nacional de Psiquiatría, Consejo Nacional Contra las Adicciones (1998), Encuesta Nacional de Adicciones, México, Secretaría de Salud-Instituto Nacional de Estadística, Geografía e Informática.

Secretaría de Salud, Instituto Nacional de Estadística, Geografía e Informática (2002), Encuesta Nacional de Adicciones, México, Secretaría de Salud-Instituto Nacional de Estadística, Geografía e Informática.

Sood, B., V. Delaney-Black, C. Covington, B. NordstromKlee, J. Ager, T. Templin, J. Janisse, S. Martier y R. J. Sokol (200I), "Prenatal Alcohol Exposure and Childhood Behavior at Age 6 to 7 years: I. Dose-Response Effect", Pediatrics. 108(2): 34.

Stratton, K., C. Howe y F. C. Battaglia (1996), "Fetal Alcohol Syndrome: Diagnosis, Epidemiology, Prevention, and Treatment", EU, The National Academies Press. Disponible en: http://books.nap.edu/catalog/499l.html.

Streissguth, A. P. (200I), "Recent Advances in Fetal Alcohol Syndrome and Alcohol Use in Pregnancy", en D. P. Agarwal y H. K. Seitz (eds.), Alcohol in Health and Disease, Nueva York, EU, Marcel Dekker, Inc., PP. 303-324.

Streissguth, A. P., Bookstein, F. L. y H. M. Barr (1996), “A dose-response study of the enduring affects of prenatal alcohol exposure, birth to I 4 years", en H. L. Spohr y H. C. Steinhausen (eds.), Alcohol, Pregnancy and the Developing Child: Fetal Alcohol Syndrome, Nueva York, EU, Cambridge University Press, PP. I4I-I68. 
Streissguth, A. P. y K. O'Malley (2000), “Neuropsychiatric Bibliografía implications and Long-term consequences of Fetal Alcohol Spectrum Disorders", Seminars in Clinical Neuropsychiatric, 5(3): 177-190.

Willford, J. A., G. A. Richardson, S. L. Leech y N. L. Day (2004), "Verbal and visuospatial learning and memory function in children with moderate prenatal alcohol exposure", Alcoholism Clinical and Experimental Research, 28(3): 497-507. 